\title{
Global Existence and Long-Time Behavior of Solutions to the Full Compressible Euler Equations with Damping and Heat Conduction in $\mathbb{R}^{3}$
}

\author{
Yunshun Wu, ${ }^{1,2}$ Yong Wang $\mathbb{D}^{3}$ and Rong Shen ${ }^{3}$ \\ ${ }^{1}$ School of Mathematical Sciences, Guizhou Normal University, Guiyang, Guizhou 550001, China \\ ${ }^{2}$ School of Mathematical Sciences and Fujian Provincial Key Laboratory on Mathematical Modeling \& High Performance \\ Scientific Computing, Xiamen University, Xiamen, Fujian 361005, China \\ ${ }^{3}$ South China Research Center for Applied Mathematics and Interdisciplinary Studies, School of Mathematical Sciences, South China \\ Normal University, Guangzhou 510631, China
}

Correspondence should be addressed to Yong Wang; wangyongxmu@163.com

Received 21 February 2021; Revised 7 April 2021; Accepted 19 April 2021; Published 11 May 2021

Academic Editor: Giuseppe Pellicane

Copyright (C) 2021 Yunshun Wu et al. This is an open access article distributed under the Creative Commons Attribution License, which permits unrestricted use, distribution, and reproduction in any medium, provided the original work is properly cited.

We study the Cauchy problem of the three-dimensional full compressible Euler equations with damping and heat conduction. We prove the existence and uniqueness of the global small $H^{N}(N \geq 3)$ solution; in particular, we only require that the $H^{4}$ norms of the initial data be small when $N \geq 5$. Moreover, we use a pure energy method to show that the global solution converges to the constant equilibrium state with an optimal algebraic decay rate as time goes to infinity.

\section{Introduction}

We study the following Cauchy problem of the full compressible Euler equations with damping and heat conduction: for $(x, t) \in \mathbb{R}^{3} \times[0, \infty)$,

$$
\left\{\begin{array}{l}
\rho_{t}+\operatorname{div}(\rho u)=0, \\
(\rho u)_{t}+\operatorname{div}(\rho u \otimes u)+\nabla P=-\alpha \rho u, \\
(\rho \mathscr{E})_{t}+\operatorname{div}(\rho u \mathscr{C}+u P)=-\alpha \rho u^{2}+\kappa \Delta \theta, \\
\left.(\rho, u, \theta)(x, t)\right|_{t=0}=\left(\rho_{0}, u_{0}, \theta_{0}\right)(x) \longrightarrow(1,0,1),|x| \longrightarrow \infty .
\end{array}\right.
$$

Here, the unknown variables $\rho=\rho(x, t), u=u(x, t)$, $\theta=\theta(x, t), P=P(x, t)$ denote the density, the velocity, the absolute temperature, and the pressure, respectively. The total energy per unit mass $\mathscr{E}=(1 / 2)|u|^{2}+e$, and $e$ is the internal energy per unit mass. The constants $\alpha>0$ and $\kappa>$ 0 are the friction damping coefficient and the thermal conductivity, respectively.
The system (1) can be used to model a compressible gas flow through a porous medium [1-3]. Assume that the gas is perfect and polytropic, then

$$
P=R \rho \theta=A e^{S / c_{v}} \rho^{\gamma}, e=c_{v} \theta, \quad c_{v}=\frac{R}{\gamma-1},
$$

where $S$ is the entropy, $R>0$ and $A>0$ are the universal gas constants, $\gamma>1$ is the adiabatic exponent, and $c_{v}>0$ is the specific heat at constant volume.

We review the known results about the compressible Euler equations with damping. There are a lot of research works on the compressible isentropic Euler equations with damping in dimension one. For the Cauchy problems, readers can refer to $[4,5]$ for the existence of the global $B V$ solutions, to [6-11] for the global $L^{\infty}$ entropy-weak solutions with vacuum, and to $[12,13]$ for small smooth solutions. For the initial-boundary value problems, readers can refer to [14, 15] for the existence of the global $L^{\infty}$ entropy-weak solutions and to $[2,16,17]$ for small smooth solutions. For the asymptotic convergence of solutions, we refer to [8-11] for $L^{\infty}$ 
entropy-weak solutions and to [13, 18-20] for small smooth solutions. In addition, there are some results on the $1 D$ compressible nonisentropic Euler equations with damping (see $[1-3,21-23])$. The global existence and long-time behavior of solutions to the multidimensional compressible isentropic Euler equations with damping were studied by many researchers (cf. [15, 24-35] and the references cited therein). Recently, the free boundary problem of the Euler equations with damping was considered (cf. [36-38]).

To the best of our knowledge, there are few results on the three-dimensional full compressible damped Euler equations (1). We first notice that the system (1) can be equivalently reduced to the $(\rho, u, \theta)$-system

$$
\left\{\begin{array}{l}
\rho_{t}+\operatorname{div}(\rho u)=0, \\
u_{t}+u \cdot \nabla u+R \theta \frac{\nabla \rho}{\rho}+R \nabla \theta=-\alpha u, \\
c_{v} \theta_{t}+c_{v} u \cdot \nabla \theta+R \theta \operatorname{div} u=\kappa \frac{\Delta \theta}{\rho},
\end{array}\right.
$$

or the $(P, u, S)$-system

$$
\left\{\begin{array}{l}
P_{t}+u \cdot \nabla P+\frac{c_{v}+R}{c_{v}} P \operatorname{div} u=\frac{R \kappa}{c_{v}} \Delta \theta, \\
u_{t}+u \cdot \nabla u+\frac{\nabla P}{\rho}=-\alpha u, \\
S_{t}+u \cdot \nabla S=R \kappa \frac{\Delta \theta}{P}
\end{array}\right.
$$

where $\rho$ and $\theta$ are given by

$\rho=A^{-\left(c_{v} /\left(c_{v}+R\right)\right)} P^{c_{v} /\left(c_{v}+R\right)} e^{-\left(S /\left(c_{v}+R\right)\right)}, \quad \theta=R^{-1} A^{c_{v} /\left(c_{v}+R\right)} P^{R /\left(c_{v}+R\right)} e^{S /\left(c_{v}+R\right)}$.

When $\kappa>0$, Chen et al. [39] considered the $(\rho, u, \theta)$ -system (3) and then used Fourier analysis methods together with energy methods to prove the global existence and timedecay rates of small smooth solutions. For the case of $\kappa=0$, the temperature equation in (3) has no dissipation, and thus, the method used in [39] is not applicable. To overcome the difficulties arising from the nondissipation of $\theta$, the researchers in $[40,41]$ studied the $(P, u, S)$-system (4) with $\kappa$ $=0$ and thus proved the similar results as the case of $\kappa>0$. An important observation is that the linear parts of $(P, u)$ and $S$ are decoupled in the linearized $(P, u, S)$-system, which helps to derive the desired estimates as done in [40, 41]. With regard to the corresponding initial-boundary value problem for $\kappa=0$ in a bounded domain, Zhang and $\mathrm{Wu}$ [42] and $\mathrm{Wu}$ [43] independently obtained the global existence and the exponential stability of small smooth solutions.

In the present paper, we shall choose the $(\rho, u, \theta)$-system and prove the global existence and uniqueness of the smooth solution to the Cauchy problem (1) near a constant equilibrium state $(1,0,1)$ for the initial data with various regularities. At the same time, we will use a pure energy method developed in $[29,44]$ to derive the optimal time-decay rates of solutions as well as their spatial derivatives of any order. Compared with the Fourier analysis method used in [39], the pure energy method can be used to obtain the optimal time-decay rates under the weak regularity assumptions, which can be seen from $L^{p} \subset \dot{H}^{-s}(1<p \leq 2,0 \leq s<3 / 2)$ or $L^{p} \subset \dot{B}_{2, \infty}^{-s}(1 \leq p<2,0<s \leq 3 / 2)$. As a byproduct, we give the optimal $L^{p}-L^{q}$-type decay rates of solutions (see Corollary 3 ).

Notation. Throughout this paper, $\nabla^{k}$ with an integer $k \geq 0$ represents the usual any spatial derivatives of order $k$. When $k<0$ or $k$ is not a positive integer, $\nabla^{k}$ means $\Lambda^{k}$ defined by $\Lambda^{k} f:=\mathscr{F}^{-1}\left(|\xi|^{k} \mathscr{F} f\right)$, where $\mathscr{F}$ is the usual Fourier transform operator and $\mathscr{F}^{-1}$ is its inverse. We denote by $L^{p}\left(\mathbb{R}^{3}\right)(1 \leq p$ $\leq \infty)$ the usual Lebesgue spaces with the norm $\|\cdot\|_{L^{p}}$. For simplicity, we write $\|\cdot\|=\|\cdot\|_{L^{2}}$. We use $H^{N}\left(\mathbb{R}^{3}\right)$ for some integer $N \geq 0$ to denote the usual Sobolev spaces with the norm $\|\cdot\|_{H^{N}}$. We use $\dot{H}^{s}\left(\mathbb{R}^{3}\right)(s \in \mathbb{R})$ to denote the homogeneous Sobolev spaces with the norm $\|\cdot\|_{\dot{H}^{s}}$ defined by $\|f\|_{\dot{H}^{s}}:=\| \nabla^{s}$ $f \|$. It is clear for $H^{0}=\dot{H}^{0}=L^{2}$.

We review the homogeneous Besov spaces. Let $\phi \epsilon$ $C_{0}^{\infty}\left(\mathbb{R}_{\xi}^{3}\right)$ satisfy $\phi(\xi)=1$ if $|\xi| \leq 1$ and $\phi(\xi)=0$ if $|\xi| \geq 2$. Define $\varphi(\xi):=\phi(\xi)-\phi(2 \xi)$ and $\varphi_{j}(\xi):=\varphi\left(2^{-j} \xi\right)$ for $j \in \mathbb{Z}$. Then, $\sum_{j \in \mathbb{Z}} \varphi_{j}(\xi)=1$ if $\xi \neq 0$. Define $\dot{\Delta}_{j} f:=\mathscr{F}^{-1}\left(\varphi_{j}\right) * f$. For $s \in \mathbb{R}$ and $1 \leq p \leq \infty$, we denote by $\dot{B}_{p, \infty}^{s}\left(\mathbb{R}^{3}\right)$ the homogeneous Besov spaces with the norm $\|\cdot\|_{\dot{B}_{p, \infty}^{s}}$ defined by $\|f\|_{\dot{B}_{p, \infty}^{s}}:=\sup _{j \in \mathbb{Z}^{2}} 2^{s j}\left\|\dot{\Delta}_{j} f\right\|_{L^{p}}$.

We employ the notation $A \lesssim B$ to mean that $A \leq C B$ for a generic positive constant $C$. We denote $A \sim B$ if $A \lesssim B$ and $B$ $\lesssim A$. We use $C_{0}$ to denote a positive constant depending additionally on the initial data. For simplicity, we write $\|(A, B)\|_{X}:=\|A\|_{X}+\|B\|_{X}$ and $\int f:=\int_{\mathbb{R}^{3}} f d x$. The notation $C^{k}(0, T ; X)(k \geq 0)$ denotes the space of $X$-valued $k$-times continuously differentiable functions on $[0, T]$.

The main results in this paper can be stated as follows.

Theorem 1. Let $N \geq 3$ be an integer. Assume that $\left(\rho_{0}-1, u_{0}\right.$, $\left.\theta_{0}-1\right) \in H^{N}$ satisfying

$$
\left\|\left(\rho_{0}-1, u_{0}, \theta_{0}-1\right)\right\|_{H^{3}}<\delta_{0}, \quad \text { if } N=3
$$

or

$$
\left\|\left(\rho_{0}-1, u_{0}, \theta_{0}-1\right)\right\|_{H^{4}}<\delta_{0}, \quad \text { if } N \geq 4
$$

for some small constant $\delta_{0}>0$. Then, the Cauchy problem (1) admits a unique global solution $(\rho, u, \theta)(t)$ such that for all $t$ $\geq 0$ and $3 \leq \ell \leq N$,

$$
\begin{aligned}
& \|(\rho-1, u, \theta-1)(t)\|_{H^{\ell}}+\left(\int_{0}^{t}\left(\|\nabla \rho(\tau)\|_{H^{\ell-1}}^{2}+\|(u, \nabla \theta)(\tau)\|_{H^{\ell}}^{2}\right) d \tau\right)^{1 / 2} \\
& \quad \leq C\left\|\left(\rho_{0}-1, u_{0}, \theta_{0}-1\right)\right\|_{H^{\ell}} .
\end{aligned}
$$


Theorem 2. Under the assumptions of Theorem 1, if further $\left(\rho_{0}-1, u_{0}, \theta_{0}-1\right) \in \dot{H}^{-s}$ for some $s \in[0,3 / 2)$, or $\left(\rho_{0}-1, u_{0}\right.$, $\left.\theta_{0}-1\right) \in \dot{B}_{2, \infty}^{-s}$ for some $s \in(0,3 / 2]$, then for all $t \geq 0$,

$$
\left\{\begin{array}{l}
\left\|\nabla^{l}(\rho-1, \theta-1)(t)\right\| \leq C_{0}(1+t)^{-((l+s) / 2)}, \quad 0 \leq l \leq N-1, \\
\left\|\nabla^{N}(\rho-1, \theta-1)(t)\right\| \leq C_{0}(1+t)^{-((N-1+s) / 2)},
\end{array}\right.
$$

and

$$
\left\{\begin{array}{l}
\left\|\nabla^{l} u(t)\right\| \leq C_{0}(1+t)^{-((l+1+s) / 2)}, \quad 0 \leq l \leq N-2, \\
\left\|\left(\nabla^{N-1} u, \nabla^{N} u\right)(t)\right\| \leq C_{0}(1+t)^{-((N-1+s) / 2)}
\end{array}\right.
$$

By Lemma 9 and Lemmas 13 and 14, we easily obtain the following $L^{p}-L^{q}$-type decay rates.

Corollary 3. Under the assumptions of Theorem 2, if $\left(\rho_{0}-1\right.$, $\left.u_{0}, \theta_{0}-1\right) \in L^{p}$ for some $p \in[1,2]$, then for $2 \leq q \leq \infty$,

$$
\begin{cases}\left\|\nabla^{l}(\rho-1, \theta-1)(t)\right\|_{L^{q}} \leq C_{0}(1+t)^{-(3 / 2)((1 / p)-(1 / q))-(l / 2)}, & 0 \leq l \leq N-3, \\ \left\|\nabla^{l} u(t)\right\|_{L^{q}} \leq C_{0}(1+t)^{-(3 / 2)((1 / p)-(1 / q))-((l+1) / 2)}, & 0 \leq l \leq N-4, \\ \left\|\nabla^{N-3} u(t)\right\|_{L^{q}} \leq C_{0}(1+t)^{-(3 / 2)((1 / p)-(1 / q))-((2 N-5) / 4)-(1 / 2 q)} . & \end{cases}
$$

We give some remarks for Theorems 1 and 2 and Corollary 3.

Remark 4. From Theorem 1 , when $N \geq 5$, we only require that the $H^{4}$ norms of the initial density, velocity, and temperature be small, while the higher-order Sobolev norms can be arbitrarily large.

Remark 5 . We claim that the decay rates except the velocity $u$ in Theorem 2 and Corollary 3 are optimal in the sense that they are consistent with those in the linearized case.

Remark 6. By Corollary 3, we prove the optimal $L^{p}-L^{q}$-type time-decay rates without the smallness assumption on the $L^{p}(1 \leq p<2)$ norm of the initial data.

Remark 7. Compared with the decay results of the full compressible Navier-Stokes equations [44, 45]

$$
\|(\rho-1, u, \theta-1)\| \leq C_{0}(1+t)^{-(3 / 4)}
$$

the density and temperature of the full compressible damped Euler equations have the same decay rates (see (9) with $l=0$ and $s=3 / 2)$; however, the decay of the $L^{2}$ norm of the velocity is improved to $(1+t)^{-(5 / 4)}($ see $(10)$ with $l=0$ and $s=3 / 2$ ) due to the damping effect.

Remark 8 . With regard to the initial-boundary value problem of the three-dimensional full compressible damped Euler equations (1), the case of $\kappa=0$ was solved in [42, 43], and the corresponding $(P, u, S)$-system was adopted. For the case of $\kappa>0$, we believe that it is more convenient to deal with the $(\rho, u, \theta)$-system, which is a forthcoming work.

The arrangement of this paper is as follows. In Section 2, we list some useful lemmas which will be frequently used. In Section 3, we establish some refined energy estimates (see Lemmas 17-19) which help us to derive important energy estimates with the minimum derivatives counts (see Lemma 20). Then, we prove the global solution (Theorem 1) and the time-decay rates (Theorem 2) in Sections 4 and 5, respectively.

\section{Preliminaries}

In this section, we will give some lemmas which are often used in the later sections. We first recall the GagliardoNirenberg-Sobolev inequality.

Lemma 9. Let $0 \leq m, \alpha \leq l$, and $2 \leq p \leq \infty$. Then, we have

$$
\left\|\nabla^{\alpha} f\right\|_{L^{p}} \lesssim\left\|\nabla^{m} f\right\|^{1-9}\left\|\nabla^{l} f\right\|^{9}
$$

where $0 \leq \vartheta \leq 1$ and $\alpha$ satisfy

$$
\alpha+3\left(\frac{1}{2}-\frac{1}{p}\right)=m(1-\vartheta)+l \vartheta
$$

Here, we require that $0<9<1, m \leq \alpha+1$, and $l \geq \alpha+2$, when $p=\infty$.

Proof. (see [46], Theorem, p.125).

We give the commutator and product estimates.

Lemma 10. Let $m$ be an integer. Define the commutator

$$
\left[\nabla^{m}, f\right] g:=\nabla^{m}(f g)-f \nabla^{m} g
$$

Then, we have for $m \geq 1$,

$$
\left\|\left[\nabla^{m}, f\right] g\right\|_{L^{p}} \lesssim\|\nabla f\|_{L^{p_{1}}}\left\|\nabla^{m-1} g\right\|_{L^{p_{2}}}+\left\|\nabla^{m} f\right\|_{L^{p_{3}}}\|g\|_{L^{p_{4}}}
$$

and for $m \geq 0$,

$$
\left\|\nabla^{m}(f g)\right\|_{L^{p}} \lesssim\|f\|_{L^{p_{1}}}\left\|\nabla^{m} g\right\|_{L^{p_{2}}}+\left\|\nabla^{m} f\right\|_{L^{p_{3}}}\|g\|_{L^{p_{4}}},
$$

where $p, p_{1}, p_{2}, p_{3}, p_{4} \in[1, \infty]$ and $1 / p=1 / p_{1}+1 / p_{2}=1 / p_{3}$ $+1 / p_{4}$.

Proof. (refer to [47], Lemma 3.1, or [48], Lemma A.4).

The following lemma gives the convenient $L^{p}$ estimates for well-prepared functions.

Lemma 11. Assume that $\|\mathrm{Q}\|_{L^{\infty}} \leq 1$ and $\|\Theta\|_{L^{\infty}} \leq 1$. Let $g$ $(\mathrm{Q}, \Theta)$ be a smooth function of $\mathrm{Q}, \Theta$ with bounded derivatives of any order, then for any integer $k \geq 1$ and $2 \leq p \leq \infty$, 


$$
\left\|\nabla^{k}(g(\mathrm{\varrho}, \Theta))\right\|_{L^{p}} \lesssim\left\|\nabla^{k} \mathrm{\varrho}\right\|_{L^{p}}+\left\|\nabla^{k} \Theta\right\|_{L^{p}} .
$$

Proof. (see [49], Lemma A.2).

As a byproduct of Lemma 11, we immediately have the following.

Corollary 12. Assume that $\|\mathrm{Q}\|_{L^{\infty}} \leq 1$. Let $g(\mathrm{Q})$ be a smooth function of $\mathrm{Q}$ with bounded derivatives of any order, then for any integer $k \geq 1$ and $2 \leq p \leq \infty$,

$$
\left\|\nabla^{k}(g(\mathrm{\varrho}))\right\|_{L^{p}} \lesssim\left\|\nabla^{k} \mathrm{Q}\right\|_{L^{p}} .
$$

Finally, we list some useful estimates or interpolation inequalities involving negative Sobolev or Besov spaces.

Lemma 13. Let $1<p \leq 2$ and $1 / 2+s / 3=1 / p$. Then, $0 \leq s$ $<3 / 2$ and

$$
\|f\|_{\dot{H}^{-s}} \lesssim\|f\|_{L^{p}}
$$

Proof. It follows from the Hardy-Littlewood-Sobolev theorem (cf. [50], Theorem 1, p.119).

Lemma 14. Let $1 \leq p<2$ and $1 / 2+s / 3=1 / p$. Then, $0<s$ $\leq 3 / 2$ and

$$
\|f\|_{\dot{B}_{2, \infty}^{-s}} \leq\|f\|_{L^{p}}
$$

Proof. (see [51], Lemma 4.1).

Lemma 15. Let $s \geq 0$ and $l \geq 0$. Then,

$$
\left\|\nabla^{l} f\right\| \lesssim\left\|\nabla^{l+1} f\right\|^{1-\vartheta}\|f\|_{\dot{H}^{-s}}^{\vartheta}, \quad \vartheta=\frac{1}{l+s+1} .
$$

Proof. (see [44], Lemma A.4).

Lemma 16. Let $s>0$ and $l \geq 0$. Then,

$$
\left\|\nabla^{l} f\right\| \lesssim\left\|\nabla^{l+1} f\right\|^{1-\vartheta}\|f\|_{\dot{B}_{2, \infty}^{-s}}^{\vartheta}, \quad \vartheta=\frac{1}{l+s+1} .
$$

Proof. We refer to [51], Lemma 4.2, by noting that $\dot{B}_{2, p}^{-s} \subset \dot{B}_{2, q}^{-s}$ for $p \leq q$.

\section{Energy Estimates}

By a simple calculation, the Cauchy problem (1) becomes

$$
\left\{\begin{array}{l}
\rho_{t}+\operatorname{div}(\rho u)=0, \\
u_{\mathrm{t}}+u \cdot \nabla u+R \theta \frac{\nabla \rho}{\rho}+R \nabla \theta=-\alpha u, \\
c_{v} \theta_{t}+c_{v} u \cdot \nabla \theta+R \theta \operatorname{div} u=\kappa \frac{\Delta \theta}{\rho}, \\
\left.(\rho, u, \theta)\right|_{t=0}=\left(\rho_{0}, u_{0}, \theta_{0}\right) .
\end{array}\right.
$$

Without loss of generality, we assume $R=c_{v}=\alpha=\kappa=1$ and choose the constant equilibrium state $(1,0,1)$. Define the perturbations

$$
\varrho=\rho-1, \quad u=u-0, \quad \Theta=\theta-1 .
$$

Then, problem (24) is reformulated as

$$
\left\{\begin{array}{l}
\mathrm{\varrho}_{t}=-u \cdot \nabla \mathrm{\varrho}-(1+\varrho) \operatorname{div} u \\
u_{t}+u+\nabla \Theta=-u \cdot \nabla u-\frac{1+\Theta}{1+\varrho} \nabla \mathrm{\varrho}, \\
\Theta_{t}-\Delta \Theta=-u \cdot \nabla \Theta-(1+\Theta) \operatorname{div} u-\frac{\varrho}{1+\varrho} \Delta \Theta, \\
\left.(\varrho, u, \Theta)\right|_{t=0}=\left(\varrho_{0}, u_{0}, \Theta_{0}\right):=\left(\rho_{0}-1, u_{0}, \theta_{0}-1\right) .
\end{array}\right.
$$

We will derive the a priori estimates for the problem (26) by assuming that for sufficiently small $\delta>0$ and some $T>0$,

$$
\sup _{0 \leq t \leq T}\|(\varrho, u, \Theta)(t)\|_{H^{N}}<\delta
$$

where $N=3$ or 4 . By Sobolev's inequality, (27) implies

$$
\frac{1}{2} \leq 1+\varrho \leq \frac{3}{2}, \quad \frac{1}{2} \leq 1+\Theta \leq \frac{3}{2} .
$$

First, we derive the energy estimates for $(\varrho, u, \Theta)$ up to order $N-1$, which contain the dissipation estimates for $u$ and $\Theta$ up to order $N-1$ and $N$, respectively.

Lemma 17. Let $N \geq 3$ and $\delta \ll 1$. If $\sup _{0 \leq t \leq T}\|(\varrho, u, \Theta)(t)\|_{H^{3}}$ $<\delta$, then for $0 \leq k \leq N-1$,

$$
\frac{d}{d t}\left\|\nabla^{k}(\mathrm{\varrho}, u, \Theta)\right\|^{2}+\left\|\nabla^{k} u\right\|^{2}+\left\|\nabla^{k+1} \Theta\right\|^{2} \lesssim \delta\left\|\nabla^{k+1} \mathrm{\varrho}\right\|^{2}
$$

Proof. It is trivial for $k=0$. Next, we will prove (29) for $1 \leq$ $k \leq N-1$. Applying $\nabla^{k}$ to $(26)_{1},(26)_{2}$, and $(26)_{3}$ and 
multiplying the resulting identities by $\nabla^{k} \mathrm{Q}, \nabla^{k} u, \nabla^{k} \Theta$, respectively; summing them up; and then integrating over $\mathbb{R}^{3}$ by parts, we get

$$
\begin{aligned}
\frac{1}{2} \frac{d}{d t} & \left\|\nabla^{k}(\varrho, u, \Theta)\right\|^{2}+\left\|\nabla^{k} u\right\|^{2}+\left\|\nabla^{k+1} \Theta\right\|^{2} \\
= & -\int \nabla^{k} \operatorname{div}(\varrho u) \cdot \nabla^{k} \mathrm{\varrho}-\int \nabla^{k}\left(u \cdot \nabla u+\frac{\Theta-\varrho}{1+\varrho} \nabla \varrho\right) \cdot \nabla^{k} u \\
& \quad-\int \nabla^{k} \operatorname{div}(\Theta u) \cdot \nabla^{k} \Theta-\int \nabla^{k}\left(\frac{\mathrm{\varrho}}{1+\mathrm{\varrho}} \Delta \Theta\right) \cdot \nabla^{k} \Theta \\
:= & I_{1}+I_{2}+I_{3}+I_{4} .
\end{aligned}
$$

Now, we estimate the terms $I_{1}-I_{4}$. For the term $I_{1}$, by integrating by parts; Hölder's, Sobolev's, and Cauchy's inequalities; and (17) of Lemma 10, we obtain

$$
\begin{aligned}
I_{1} & =\int \nabla^{k-1} \operatorname{div}(\mathrm{\varrho} u) \cdot \nabla^{k-1} \Delta \mathrm{\varrho} \\
& \leq\left\|\nabla^{k}(\mathrm{\varrho} u)\right\|\left\|\nabla^{k+1} \mathrm{\varrho}\right\| \\
& \leq\left(\left\|\nabla^{k} \mathrm{\varrho}\right\|_{L^{6}}\|u\|_{L^{3}}+\|\mathrm{\varrho}\|_{L^{\infty}}\left\|\nabla^{k} u\right\|\right)\left\|\nabla^{k+1} \mathrm{\varrho}\right\| \\
& \leq \delta\left(\left\|\nabla^{k+1} \mathrm{\varrho}\right\|^{2}+\left\|\nabla^{k} u\right\|^{2}\right) .
\end{aligned}
$$

For the term $I_{2}$, by integrating by parts; Hölder's, Sobolev's, and Cauchy's inequalities; Lemma 10; and Corollary 12, we obtain

$$
\begin{aligned}
\int \nabla^{k}(u \cdot \nabla u) \cdot \nabla^{k} u & =\int\left(u \cdot \nabla \nabla^{k} u \cdot \nabla^{k} u+\left[\nabla^{k}, u\right] \cdot \nabla u \cdot \nabla^{k} u\right) \\
& =-\frac{1}{2} \int \operatorname{div} u\left|\nabla^{k} u\right|^{2}+\int\left[\nabla^{k}, u\right] \cdot \nabla u \cdot \nabla^{k} u \\
& \leqslant\|\operatorname{div} u\|_{L^{\infty}}\left\|\nabla^{k} u\right\|^{2}+\left\|\left[\nabla^{k}, u\right] \cdot \nabla u\right\|\left\|\nabla^{k} u\right\| \\
& \leqslant\|u\|_{H^{3}}\left\|\nabla^{k} u\right\|^{2}+\|\nabla u\|_{L^{\infty}}\left\|\nabla^{k} u\right\|^{2} \\
& \leqslant \delta\left\|\nabla^{k} u\right\|^{2}
\end{aligned}
$$

$$
\begin{aligned}
\int \nabla^{k} & \left(\frac{\Theta-\varrho}{1+\varrho} \nabla \varrho\right) \cdot \nabla^{k} u \lesssim\left\|\nabla^{k}\left(\frac{\Theta-\varrho}{1+\varrho} \nabla \varrho\right)\right\|\left\|\nabla^{k} u\right\| \\
\leq & \left(\left\|\nabla^{k}\left(\frac{\nabla \varrho}{1+\varrho} \Theta\right)\right\|+\left\|\nabla^{k}\left(\frac{\varrho}{1+\varrho} \nabla \varrho\right)\right\|\right)\left\|\nabla^{k} u\right\| \\
\leq & \left(\left\|\nabla^{k}\left(\frac{\nabla \varrho}{1+\varrho}\right)\right\|\|\Theta\|_{L^{\infty}}+\left\|\frac{\nabla \varrho}{1+\varrho}\right\|_{L^{3}}\left\|\nabla^{k} \Theta\right\|_{L^{6}}\right)\left\|\nabla^{k} u\right\| \\
& +\left(\left\|\nabla^{k}\left(\frac{\varrho}{1+\varrho}\right)\right\|_{L^{6}}\|\nabla \varrho\|_{L^{3}}+\left\|\frac{\varrho}{1+\varrho}\right\|_{L^{\infty}}\left\|\nabla^{k+1} \varrho\right\|\right)\left\|\nabla^{k} u\right\| \\
\leq & \delta\left(\left\|\nabla^{k+1}(\varrho, \Theta)\right\|^{2}+\left\|\nabla^{k} u\right\|^{2}\right) .
\end{aligned}
$$

In light of (32) and (33), we have

$$
I_{2} \leq \delta\left(\left\|\nabla^{k+1}(\varrho, \Theta)\right\|^{2}+\left\|\nabla^{k} u\right\|^{2}\right)
$$

As with the term $I_{1}$, we obtain

$$
I_{3} \lesssim \delta\left(\left\|\nabla^{k+1} \Theta\right\|^{2}+\left\|\nabla^{k} u\right\|^{2}\right) .
$$

For the term $I_{4}$, by integrating by parts; Hölder's, Sobolev's, and Young's inequalities; Lemmas 9 and 10; and Corollary 12, we obtain

$$
\begin{aligned}
& I_{4}=\int \nabla^{k-1}\left(\frac{\mathrm{e}}{1+\varrho} \Delta \Theta\right) \cdot \nabla^{k-1} \Delta \Theta \leq\left\|\nabla^{k-1}\left(\frac{\mathrm{e}}{1+\varrho} \Delta \Theta\right)\right\|\left\|\nabla^{k+1} \Theta\right\| \\
& \leq\left(\left\|\nabla^{k-1}\left(\frac{\mathrm{e}}{1+\mathrm{e}}\right)\right\|_{L^{\circ}}\|\Delta \Theta\|_{L^{3}}+\left\|\frac{\mathrm{e}}{1+\mathrm{e}}\right\|_{L^{\infty}}\left\|\nabla^{k+1} \Theta\right\|\right)\left\|\nabla^{k+1} \Theta\right\| \\
& \leq\left(\left\|\nabla^{k} \mathrm{e}\right\|\left\|\nabla^{2} \Theta\right\|_{L^{3}}+\|\boldsymbol{e}\|_{H^{2}}\left\|\nabla^{k+1} \Theta\right\|\right)\left\|\nabla^{k+1} \Theta\right\| \\
& \leq\left\|\nabla^{k+1} \mathrm{e}\right\|^{k /(k+1)}\|\Theta\|^{1 /(k+1)}\left\|\nabla^{k+1} \Theta\right\|^{1 /(k+1)}\left\|\nabla^{3(k+1) / 2 k} \Theta\right\|^{k /(k+1)}\left\|\nabla^{k+1} \Theta\right\|+\delta\left\|\nabla^{k+1} \Theta\right\|^{2} \\
& \leq\|(e, \Theta)\|_{H^{3}}\left(\left\|\nabla^{k+1} \Theta\right\|+\left\|\nabla^{k+1} \Theta\right\|\right)\left\|\nabla^{k+1} \Theta\right\|+\delta\left\|\nabla^{k+1} \Theta\right\|^{2} \\
& \leq \delta\left\|\nabla^{k+1}(\mathrm{\varrho}, \Theta)\right\|^{2} \text {. }
\end{aligned}
$$

Plugging the estimates for $I_{1}-I_{4}$ into (30), we deduce (29).

Next, we derive the $\mathrm{N}$-th-order energy estimates for $(\mathrm{\varrho}$, $u, \Theta)$, which contain the dissipation estimates for $u$ and $\Theta$ of order $N$ and $N+1$, respectively.

Lemma 18. Let $N \geq 3$. Then, we have

$$
\frac{d}{d t} \int \mathfrak{F}^{N}(t)+\left\|\nabla^{N} u\right\|^{2}+\left\|\nabla^{N+1} \Theta\right\|^{2} \lesssim \delta\left\|\nabla^{N}(\varrho, \Theta)\right\|^{2},
$$

under the assumption of

$$
\sup _{0 \leq t \leq T}\|(\varrho, u, \Theta)(t)\|_{H^{3}}<\delta \ll 1, \quad \text { if } N=3,
$$

or

$$
\sup _{0 \leq t \leq T}\|(\mathrm{\varrho}, u, \Theta)(t)\|_{H^{4}}<\delta \ll 1, \quad \text { if } N \geq 4 .
$$

Here,

$$
\begin{aligned}
\mathfrak{F}^{N}(t):= & \frac{1+\Theta}{1+\varrho}\left|\nabla^{N} \mathrm{\varrho}\right|^{2}+(1+\mathrm{\varrho})\left|\nabla^{N-1} \operatorname{div} u\right|^{2} \\
& +\left|\nabla^{N-1} \operatorname{curl} u\right|^{2}+\frac{1+\varrho}{1+\Theta}\left|\nabla^{N} \Theta\right|^{2} .
\end{aligned}
$$

Proof. We first prove (ii). For equations $(26)_{1}-(26)_{3}$, computing

$$
\begin{aligned}
\int & {\left[\frac{1+\Theta}{1+\varrho} \nabla^{N} \varrho \cdot \nabla^{N}(26)_{1}+(1+\varrho) \nabla^{N-1} \operatorname{div} u \cdot \nabla^{N-1} \operatorname{div}(26)_{2}\right.} \\
& \left.+\frac{1+\varrho}{1+\Theta} \nabla^{N} \Theta \cdot \nabla^{N}(26)_{3}\right]
\end{aligned}
$$


and integrating by parts, we have

$$
\begin{aligned}
\frac{1}{2} \frac{d}{d t} \int & {\left[\frac{1+\Theta}{1+\varrho}\left|\nabla^{N} \varrho\right|^{2}+(1+\varrho)\left|\nabla^{N-1} \operatorname{div} u\right|^{2}+\frac{1+\varrho}{1+\Theta}\left|\nabla^{N} \Theta\right|^{2}\right] } \\
& +\int\left[(1+\varrho)\left|\nabla^{N-1} \operatorname{div} u\right|^{2}+\frac{1+\varrho}{1+\Theta}\left|\nabla^{N+1} \Theta\right|^{2}\right] \\
= & \frac{1}{2} \int\left[\partial_{t}\left(\frac{1+\Theta}{1+\varrho}\right)\left|\nabla^{N} \varrho\right|^{2}+\partial_{t} \varrho\left|\nabla^{N-1} \operatorname{div} u\right|^{2}+\partial_{t}\left(\frac{1+\varrho}{1+\Theta}\right)\left|\nabla^{N} \Theta\right|^{2}\right] \\
& -\int\left\{\frac{1+\Theta}{1+\varrho} \nabla^{N} \varrho \cdot \nabla^{N}[(1+\varrho) \operatorname{div} u]\right. \\
& \left.+(1+\varrho) \nabla^{N-1} \operatorname{div} u \cdot \nabla^{N-1} \operatorname{div}\left(\frac{1+\Theta}{1+\varrho} \nabla \varrho\right)\right\} \\
& -\int\left\{(1+\varrho) \nabla^{N-1} \operatorname{div} u \cdot \nabla^{N-1} \Delta \Theta+\frac{1+\varrho}{1+\Theta} \nabla^{N} \Theta \cdot \nabla^{N}[(1+\Theta) \operatorname{div} u]\right\} \\
& -\int \nabla\left(\frac{1+\varrho}{1+\Theta}\right) \cdot \nabla^{N} \Theta \cdot \nabla^{N+1} \Theta-\int \frac{1+\Theta}{1+\varrho} \nabla^{N} \varrho \cdot \nabla^{N}(u \cdot \nabla \varrho) \\
& -\int(1+\varrho) \nabla^{N-1} \operatorname{div} u \cdot \nabla^{N-1} \operatorname{div}(u \cdot \nabla u)-\int \frac{1+\varrho}{1+\Theta} \nabla^{N} \Theta \cdot \nabla^{N}(u \cdot \nabla \Theta) \\
& -\int \frac{1+\varrho}{1+\Theta} \nabla^{N} \Theta \cdot \nabla^{N}\left(\frac{\varrho}{1+\varrho} \Delta \Theta\right) \\
:= & \sum_{i=1}^{8} J_{i} .
\end{aligned}
$$

Now, we estimate the terms $J_{1}-J_{8}$. By $(26)_{1},(26)_{3},(28)$, and Hölder's and Sobolev's inequalities, we have

$$
\begin{aligned}
J_{1} & \lesssim\left(\left\|\Theta_{t}\right\|_{L^{\infty}}+\left\|\mathrm{\varrho}_{t}\right\|_{L^{\infty}}\right)\left\|\nabla^{N}(\varrho, u, \Theta)\right\|^{2} \\
& \lesssim\left(\|(\varrho, u)\|_{H^{3}}+\|\Theta\|_{H^{4}}\right)\left\|\nabla^{N}(\varrho, u, \Theta)\right\|^{2} \\
& \lesssim \delta\left\|\nabla^{N}(\varrho, u, \Theta)\right\|^{2}
\end{aligned}
$$

By the commutator notation (15), the commutator estimate (16), integrating by parts, and Lemma 11, we have

$$
\begin{aligned}
J_{2}= & -\int\left[(1+\Theta) \nabla^{N} \varrho \cdot \nabla^{N} \operatorname{div} u+(1+\Theta) \nabla^{N-1} \operatorname{div} u \cdot \nabla^{N-1} \Delta \mathrm{\varrho}\right] \\
& -\int\left\{\frac{1+\Theta}{1+\rho} \nabla^{N} \varrho \cdot\left[\nabla^{N}, 1+\varrho\right] \operatorname{div} u+(1+\varrho) \nabla^{N-1} \operatorname{div} u\right. \\
& \left.\cdot\left[\nabla^{N-1} \operatorname{div}, \frac{1+\Theta}{1+\varrho}\right] \nabla \varrho\right\} \\
\lesssim & \int\left|\nabla \Theta\left\|\nabla^{N} \varrho\right\| \nabla^{N} u\right|+\int \mid \nabla^{N} \varrho \|\left[\nabla^{N}, 1\right. \\
& +\varrho] \nabla u\left|+\int\right| \nabla^{N} u||\left[\nabla^{N}, \frac{1+\Theta}{1+\varrho}\right] \nabla \varrho \mid \\
\lesssim & \|\nabla \Theta\|_{L^{\infty}}\left\|\nabla^{N} \varrho\right\|\left\|\nabla^{N} u\right\|+\left\|\nabla^{N} \varrho\right\| \|\left[\nabla^{N}, 1\right. \\
& +\varrho] \nabla u\|+\| \nabla^{N} u\|\|\left[\nabla^{N}, \frac{1+\Theta}{1+\varrho}\right] \nabla \varrho \| \\
\lesssim & \|\nabla \Theta\|_{L^{\infty}}\left\|\nabla^{N} u\right\|\left\|\nabla^{N} \varrho\right\|+\left\|\nabla^{N} \varrho\right\|\left(\|\nabla \varrho\|_{L^{\infty}}\left\|\nabla^{N} u\right\|\right. \\
& \left.+\left\|\nabla^{N} \varrho\right\|\|\nabla u\|_{L^{\infty}}\right)+\left\|\nabla^{N} u\right\|\left(\left\|\nabla\left(\frac{1+\Theta}{1+\varrho}\right)\right\|\left\|_{L^{\infty}}\right\| \nabla^{N} \varrho \|\right. \\
& \left.+\left\|\nabla^{N}\left(\frac{1+\Theta}{1+\varrho}\right)\right\|\|\nabla \varrho\|_{L^{\infty}}\right) \\
\leqslant & \delta\left\|\nabla^{N}(\varrho, u, \Theta)\right\|^{2},
\end{aligned}
$$

$$
\begin{aligned}
J_{3}= & -\int(1+\varrho) \nabla^{N-1} \operatorname{div} u \cdot \nabla^{N-1} \Delta \Theta+(1+\varrho) \nabla^{N} \Theta \cdot \nabla^{N} \operatorname{div} u \\
& -\int \frac{1+\varrho}{1+\Theta} \nabla^{N} \Theta \cdot\left[\nabla^{N}, 1+\Theta\right] \operatorname{div} u \\
\lesssim & \int|\nabla \varrho|\left|\nabla^{N} \Theta\right|\left|\nabla^{N-1} \operatorname{div} u\right| \\
& +\int\left|\nabla^{N} \Theta \|\left[\nabla^{N}, 1+\Theta\right] \nabla u\right| \\
\lesssim & \|\nabla \varrho\|_{L^{\infty}}\left\|\nabla^{N} \Theta\right\|\left\|\nabla^{N} u\right\| \\
& +\left\|\nabla^{N} \Theta\right\|\left(\|\nabla \Theta\|_{L^{\infty}}\left\|\nabla^{N} u\right\|+\left\|\nabla^{N} \Theta\right\|\|\nabla u\|_{L^{\infty}}\right) \\
\lesssim & \delta\left\|\nabla^{N}(u, \Theta)\right\|^{2} .
\end{aligned}
$$

By Hölder's, Sobolev's, and Cauchy's inequalities and Lemma 11, we obtain

$$
J_{4} \lesssim\left\|\nabla\left(\frac{1+\varrho}{1+\Theta}\right)\right\|_{L^{3}}\left\|\nabla^{N} \Theta\right\|_{L^{6}}\left\|\nabla^{N+1} \Theta\right\| \lesssim \delta\left\|\nabla^{N+1} \Theta\right\|^{2} .
$$

By Lemma 10, integrating by parts, and Hölder's, Sobolev's, and Cauchy's inequalities, we have

$$
\begin{aligned}
J_{5} & =-\int \frac{1+\Theta}{1+\varrho} \nabla^{N} \varrho \cdot \nabla^{N}(u \cdot \nabla \varrho) \\
& =-\frac{1}{2} \int \frac{1+\Theta}{1+\varrho} u \cdot \nabla\left|\nabla^{N} \varrho\right|^{2}-\int \frac{1+\Theta}{1+\varrho} \nabla^{N} \varrho \cdot\left[\nabla^{N}, u\right] \cdot \nabla \varrho \\
& =\frac{1}{2} \int \operatorname{div}\left(\frac{1+\Theta}{1+\varrho} u\right)\left|\nabla^{N} \varrho\right|^{2}-\int \frac{1+\Theta}{1+\varrho} \nabla^{N} \varrho \cdot\left[\nabla^{N}, u\right] \cdot \nabla \varrho \\
& \leq \delta\left\|\nabla^{N} \varrho\right\|^{2}+\left\|\nabla^{N} \varrho\right\|\left\|\left[\nabla^{N}, u\right] \cdot \nabla \varrho\right\| \\
& \leq \delta\left\|\nabla^{N} \varrho\right\|^{2}+\left\|\nabla^{N} \varrho\right\|\left(\|\nabla u\|_{L^{\infty}}\left\|\nabla^{N} \varrho\right\|+\left\|\nabla^{N} u\right\|\|\nabla \varrho\|_{L^{\infty}}\right) \\
& \leq \delta\left\|\nabla^{N}(\varrho, u)\right\|^{2} .
\end{aligned}
$$

Note that

$$
\operatorname{div}(u \cdot \nabla u)=\nabla u:(\nabla u)^{T}+u \cdot \nabla \operatorname{div} u
$$

where the double dots : mean that $\mathbb{A}: \mathbb{B}=\sum_{i, j=1}^{3} a_{i j} b_{i j}$ for two $3 \times 3$ matrices $\mathbb{A}=\left(a_{i j}\right)$ and $\mathbb{B}=\left(b_{i j}\right)$. By Hölder's, Sobolev's, and Cauchy's inequalities and Lemma 10, we estimate

$$
\begin{aligned}
J_{6}= & -\int(1+\varrho) \nabla^{N-1} \operatorname{div} u \cdot \nabla^{N-1} \operatorname{div}(u \cdot \nabla u) \\
= & -\int(1+\varrho) \nabla^{N-1} \operatorname{div} u \cdot \nabla^{N-1}\left[\nabla u:(\nabla u)^{T}+u \cdot \nabla \operatorname{div} u\right] \\
= & -\int(1+\varrho) \nabla^{N-1} \operatorname{div} u \cdot \nabla^{N-1}\left[\nabla u:(\nabla u)^{T}\right] \\
& -\int(1+\varrho) \nabla^{N-1} \operatorname{div} u \cdot \nabla^{N-1}(u \cdot \nabla \operatorname{div} u)
\end{aligned}
$$




$$
\begin{aligned}
& =-\int(1+\mathrm{Q}) \nabla^{N-1} \operatorname{div} u \cdot \nabla^{N-1}\left[\nabla u:(\nabla u)^{T}\right] \\
& -\frac{1}{2} \int(1+\mathrm{e}) u \cdot \nabla\left|\nabla^{N-1} \operatorname{div} u\right|^{2} \\
& -\int(1+\varrho) \nabla^{N-1} \operatorname{div} u \cdot\left[\nabla^{N-1}, u\right] \cdot \nabla \operatorname{div} u \\
& =-\int(1+\mathrm{Q}) \nabla^{N-1} \operatorname{div} u \cdot \nabla^{N-1}\left[\nabla u:(\nabla u)^{T}\right] \\
& +\frac{1}{2} \int \operatorname{div}[(1+\varrho) u]\left|\nabla^{N-1} \operatorname{div} u\right|^{2} \\
& -\int(1+\mathrm{Q}) \nabla^{N-1} \operatorname{div} u \cdot\left[\nabla^{N-1}, u\right] \cdot \nabla \operatorname{div} u \\
& \lesssim\left\|\nabla^{N-1} \operatorname{div} u\right\|\left\|\nabla^{N-1}\left[\nabla u:(\nabla u)^{T}\right]\right\| \\
& +\|\operatorname{div}[(1+\varrho) u]\|_{L^{\infty}}\left\|\nabla^{N-1} \operatorname{div} u\right\|^{2} \\
& +\left\|\nabla^{N-1} \operatorname{div} u\right\|\left\|\left[\nabla^{N-1}, u\right] \cdot \nabla \operatorname{div} u\right\| \\
& \lesssim\left\|\nabla^{N} u\right\|\|\nabla u\|_{L^{\infty}}\left\|\nabla^{N} u\right\|+\delta\left\|\nabla^{N} u\right\|^{2} \\
& +\left\|\nabla^{N} u\right\|\left(\left\|\nabla^{N-1} u\right\|_{L^{6}}\|\nabla \operatorname{div} u\|_{L^{3}}+\|\nabla u\|_{L^{\infty}}\left\|\nabla^{N-1} \operatorname{div} u\right\|\right) \\
& \lesssim \delta\left\|\nabla^{N} u\right\|^{2} \text {, } \\
& J_{7}=-\int \frac{1+\varrho}{1+\Theta} \nabla^{N} \Theta \cdot \nabla^{N}(u \cdot \nabla \Theta) \\
& \lesssim\left\|\nabla^{N} \Theta\right\|\left\|\nabla^{N}(u \cdot \nabla \Theta)\right\| \\
& \lesssim\left\|\nabla^{N} \Theta\right\|\left(\|u\|_{L^{\infty}}\left\|\nabla^{N+1} \Theta\right\|+\left\|\nabla^{N} u\right\|\|\nabla \Theta\|_{L^{\infty}}\right) \\
& \leq \delta\left(\left\|\nabla^{N}(u, \Theta)\right\|^{2}+\left\|\nabla^{N+1} \Theta\right\|^{2}\right) \text {. }
\end{aligned}
$$

By integrating by parts and by the product estimates (17) of Lemma 10 and Corollary 12, we have

$$
\begin{aligned}
J_{8}= & -\int \frac{1+\varrho}{1+\Theta} \nabla^{N} \Theta \cdot \nabla^{N}\left(\frac{\varrho}{1+\varrho} \Delta \Theta\right) \\
= & \int \operatorname{div}\left(\frac{1+\varrho}{1+\Theta} \nabla^{N} \Theta\right) \cdot \nabla^{N-1}\left(\frac{\varrho}{1+\varrho} \Delta \Theta\right) \\
= & \int\left[\nabla\left(\frac{1+\varrho}{1+\Theta}\right) \nabla^{N} \Theta+\frac{1+\varrho}{1+\Theta} \nabla^{N+1} \Theta\right] \cdot \nabla^{N-1}\left(\frac{\varrho}{1+\varrho} \Delta \Theta\right) \\
\leq & \left(\left\|\nabla\left(\frac{1+\varrho}{1+\Theta}\right)\right\|\left\|_{L^{3}}\right\| \nabla^{N} \Theta\left\|_{L^{6}}+\right\| \nabla^{N+1} \Theta \|\right)\left\|\nabla^{N-1}\left(\frac{\varrho}{1+\varrho} \Delta \Theta\right)\right\| \\
\leq & \left(\left\|\nabla\left(\frac{1+\varrho}{1+\Theta}\right)\right\|\left\|_{L^{3}}\right\| \nabla^{N} \Theta\left\|_{L^{6}}+\right\| \nabla^{N+1} \Theta \|\right) \\
& \cdot\left(\left\|\nabla^{N-1}\left(\frac{\varrho}{1+\varrho}\right)\right\|\|\Delta \Theta\|_{L^{3}}+\|\varrho\|_{L^{\infty}}\left\|\nabla^{N+1} \Theta\right\|\right) \\
\leq & \left(\|(\varrho, \Theta)\|_{H^{3}}\left\|\nabla^{N+1} \Theta\right\|+\left\|\nabla^{N+1} \Theta\right\|\right)\left\|\nabla^{N} \varrho\right\|\|\Theta\|_{H^{3}}+\|\varrho\|_{H^{3}}\left\|\nabla^{N+1} \Theta\right\| \\
\leq & \delta\left(\left\|\nabla^{N} \varrho\right\|^{2}+\left\|\nabla^{N+1} \Theta\right\|^{2}\right) .
\end{aligned}
$$

Plugging the estimates for $J_{1}-J_{8}$ into (42), by (28), since $\delta$ is small, we deduce

$$
\begin{aligned}
\frac{d}{d t} \int & {\left[\frac{1+\Theta}{1+\varrho}\left|\nabla^{N} \mathrm{\varrho}\right|^{2}+(1+\mathrm{\varrho})\left|\nabla^{N-1} \operatorname{div} u\right|^{2}+\frac{1+\varrho}{1+\Theta}\left|\nabla^{N} \Theta\right|^{2}\right] } \\
& +\left\|\nabla^{N-1} \operatorname{div} u\right\|^{2}+\left\|\nabla^{N+1} \Theta\right\|^{2} \\
\leq & \left\|\nabla^{N}(\mathrm{\varrho}, u, \Theta)\right\|^{2} .
\end{aligned}
$$

Rewrite $(26)_{2}$ as

$$
u_{t}+u=-\nabla \mathrm{\varrho}-\nabla \Theta-u \cdot \nabla u+\frac{\varrho-\Theta}{1+\varrho} \nabla \mathrm{\varrho} .
$$

Applying curl to (51), we obtain

$$
(\operatorname{curl} u)_{t}+\operatorname{curl} u=-\operatorname{curl}(u \cdot \nabla u)+\nabla\left(\frac{\varrho-\Theta}{1+\varrho}\right) \times \nabla \mathrm{\varrho},
$$

where $\times$ represents the cross product of vectors. Applying $\nabla^{N-1}$ to (52), multiplying the resulting identity by $\nabla^{N-1}$ curl $u$ and integrating over $\mathbb{R}^{3}$, we obtain

$$
\begin{aligned}
& \frac{1}{2} \frac{d}{d t} \int\left|\nabla^{N-1} \operatorname{curl} u\right|^{2}+\left\|\nabla^{N-1} \operatorname{curl} u\right\|^{2} \\
= & -\int \nabla^{N-1} \operatorname{curl}(u \cdot \nabla u) \cdot \nabla^{N-1} \operatorname{curl} u \\
& +\int \nabla^{N-1}\left[\nabla\left(\frac{\varrho-\Theta}{1+\varrho}\right) \times \nabla \mathrm{\varrho}\right] \cdot \nabla^{N-1} \operatorname{curl} u \\
:= & J_{9}+J_{10} .
\end{aligned}
$$

By Hölder's, Sobolev's, and Cauchy's inequalities and Lemmas 10 and 11, we estimate

$$
\begin{aligned}
J_{9}= & -\int \nabla^{N-1} \operatorname{curl}(u \cdot \nabla u) \cdot \nabla^{N-1} \operatorname{curl} u \\
= & -\frac{1}{2} \int u \cdot \nabla\left|\nabla^{N-1} \operatorname{curl} u\right|^{2}-\int\left[\nabla^{N-1} \operatorname{curl}, u\right] \cdot \nabla u \cdot \nabla^{N-1} \operatorname{curl} u \\
= & \left.\frac{1}{2} \int \operatorname{div} u \nabla^{N-1} \operatorname{curl} u\right|^{2}-\int\left[\nabla^{N-1} \operatorname{curl}, u\right] \cdot \nabla u \cdot \nabla^{N-1} \operatorname{curl} u \\
& \leq \delta\left\|\nabla^{N-1} \operatorname{curl} u\right\|^{2}+\int\left|\left[\nabla^{N}, u\right] \cdot \nabla u \| \nabla^{N} u\right| \\
& \leq \delta\left\|\nabla^{N-1} \operatorname{curl} u\right\|^{2}+\left\|\left[\nabla^{N}, u\right] \cdot \nabla u\right\|\left\|\nabla^{N} u\right\| \\
& \leq \delta\left\|\nabla^{N-1} \operatorname{curl} u\right\|^{2}+\|\nabla u\|_{L^{\infty}}\left\|\nabla^{N} u\right\|^{2} \leq \delta\left\|\nabla^{N} u\right\|^{2} \\
J_{10} & =\int \nabla^{N-1}\left[\nabla\left(\frac{\varrho-\Theta}{1+\varrho}\right) \times \nabla \varrho\right] \cdot \nabla^{N-1} \operatorname{curl} u \\
\leq & \left\|\nabla^{N-1}\left[\nabla\left(\frac{\varrho-\Theta}{1+\varrho}\right) \times \nabla \varrho\right]\right\|\left\|\nabla^{N-1} \operatorname{curl} u\right\| \\
\leq & \left(\left\|\nabla^{N}\left(\frac{\varrho-\Theta}{1+\varrho}\right)\right\|\|\nabla \varrho\|_{L^{\infty}}\right. \\
& \left.+\left\|\nabla\left(\frac{\varrho-\Theta}{1+\varrho}\right)\right\|_{L^{\infty}}\left\|\nabla^{N} \mathrm{\varrho}\right\|\right)\left\|\nabla^{N-1} \operatorname{curl} u\right\| \\
& \leq\left(\left\|\nabla^{N}(\varrho, \Theta)\right\|\|\varrho\|_{H^{3}}+\|(\varrho, \Theta)\|_{H^{3}}\left\|\nabla^{N} \mathrm{\varrho}\right\|\right)\left\|\nabla^{N} u\right\| \\
\leq & \left\|\nabla^{N}(\varrho, u, \Theta)\right\|^{2} .
\end{aligned}
$$


Plugging the estimates for $J_{9}-J_{10}$ into (53), we obtain

$$
\frac{d}{d t} \int\left|\nabla^{N-1} \operatorname{curl} u\right|^{2}+\left\|\nabla^{N-1} \operatorname{curl} u\right\|^{2} \leqslant \delta\left\|\nabla^{N}(\varrho, u, \Theta)\right\|^{2} .
$$

Adding (55) to (50), noting

$$
\left\|\nabla^{N} u\right\|^{2}=\left\|\nabla^{N-1} \operatorname{div} u\right\|^{2}+\left\|\nabla^{N-1} \operatorname{curl} u\right\|^{2},
$$

since $\delta$ is small, we deduce (37).

Now, we prove (i). Note that all the estimates for $J_{2}-J_{10}$ in the proof of (ii) also hold under the assumptions of $N=$ 3 and $\sup _{0 \leq t \leq T}\|(\mathrm{Q}, u, \Theta)(t)\|_{H^{3}}<\delta$. Next, we only need to estimate the term $J_{1}$ for $N=3$ under the condition of $\sup _{0 \leq t \leq T}\|(\varrho, u, \Theta)(t)\|_{H^{3}}<\delta$. Note that from $(26)_{1,2}$

$$
\begin{aligned}
& \mathrm{\varrho}_{t}=-\mathrm{\varrho} \operatorname{div} u-\operatorname{div} u-u \cdot \nabla \mathrm{Q}, \\
& \Theta_{t}=-u \cdot \nabla \Theta-\operatorname{div} u-\Theta \operatorname{div} u+\frac{1}{1+\varrho} \Delta \Theta .
\end{aligned}
$$

For $N=3$, if $\sup _{0 \leq t \leq T}\|(\mathrm{\varrho}, u, \Theta)(t)\|_{H^{3}}<\delta$, then we can estimate

$$
\begin{aligned}
J_{1}= & \frac{1}{2} \int\left[\partial_{t}\left(\frac{1+\Theta}{1+\varrho}\right)\left|\nabla^{3} \mathrm{\varrho}\right|^{2}+\partial_{t} \varrho\left|\nabla^{2} \operatorname{div} u\right|^{2}+\partial_{t}\left(\frac{1+\varrho}{1+\Theta}\right)\left|\nabla^{3} \Theta\right|^{2}\right] \\
\lesssim & \left(\left\|\Theta_{t}\right\|_{L^{\infty}}+\left\|\varrho_{t}\right\|_{L^{\infty}}\right)\left\|\nabla^{3}(\varrho, u, \Theta)\right\|^{2} \\
\lesssim & \left(\|\varrho\|_{L^{\infty}}\|\nabla u\|_{L^{\infty}}+\|\nabla u\|_{L^{\infty}}+\|u\|_{L^{\infty}}\|\nabla \mathrm{\varrho}\|_{L^{\infty}}\right)\left\|\nabla^{3}(\varrho, u, \Theta)\right\|^{2} \\
& +\left(\|u\|_{L^{\infty}}\|\nabla \Theta\|_{L^{\infty}}+\|\nabla u\|_{L^{\infty}}\right. \\
& \left.+\|\Theta\|_{L^{\infty}}\|\nabla u\|_{L^{\infty}}\right)\left\|\nabla^{3}(\varrho, u, \Theta)\right\|^{2} \\
& +\left\|\nabla^{2} \Theta\right\|_{L^{\infty}}\left\|\nabla^{3}(\varrho, u, \Theta)\right\|^{2} \\
\lesssim & \delta\left\|\nabla^{3}(\varrho, u, \Theta)\right\|^{2}+\left\|\nabla^{2} \Theta\right\|_{L^{\infty}}\left\|\nabla^{3}(\varrho, u, \Theta)\right\|^{2} \\
\lesssim & \delta\left(\left\|\nabla^{3}(\varrho, u, \Theta)\right\|^{2}+\left\|\nabla^{2} \Theta\right\|_{L^{\infty}}^{2}\right) \\
\lesssim & \delta\left(\left\|\nabla^{3}(\varrho, u, \Theta)\right\|^{2}+\left\|\nabla^{4} \Theta\right\|^{2}\right),
\end{aligned}
$$

where we have used the interpolation estimate

$$
\left\|\nabla^{2} \Theta\right\|_{L^{\infty}} \lesssim\left\|\nabla^{3} \Theta\right\|^{1 / 2}\left\|\nabla^{4} \Theta\right\|^{1 / 2}
$$

Thus, combining the new estimate (58) with the estimates for $J_{2}-J_{10}$ with $N=3$, we can deduce from (42) that (37) holds for $N=3$.

We shall derive the dissipation estimates for e up to order $N$.
Lemma 19. Let $N \geq 3$ and $\delta \ll 1$. If $\sup _{0 \leq t \leq T}\|(\varrho, u, \Theta)(t)\|_{H^{3}}$ $<\delta$, then for $0 \leq k \leq N-1$,

$$
\frac{d}{d t} \int \nabla^{k} u \cdot \nabla \nabla^{k} \mathrm{Q}+\left\|\nabla^{k+1} \mathrm{Q}\right\|^{2} \lesssim\left\|\nabla^{k} u\right\|^{2}+\left\|\nabla^{k+1}(u, \Theta)\right\|^{2} .
$$

Proof. Rewrite $(26)_{2}$ as

$$
\nabla \mathrm{\varrho}=-u_{t}-u-\nabla \Theta-u \cdot \nabla u+\frac{\varrho-\Theta}{1+\varrho} \nabla \varrho .
$$

Applying $\nabla^{k}$ to (61) and multiplying the resulting identity by $\nabla \nabla^{k}$ and then integrating over $\mathbb{R}^{3}$ by parts and by Hölder's and Cauchy's inequalities, we have

$$
\begin{aligned}
& \left\|\nabla^{k+1} \varrho\right\|^{2} \leq-\int \partial_{t} \nabla^{k} u \cdot \nabla \nabla^{k} \mathrm{\varrho}+\left\|\nabla^{k} u\right\|^{2} \\
& +\left\|\nabla^{k+1} \Theta\right\|^{2}+\left\|\nabla^{k}(u \cdot \nabla u)\right\|^{2}+\left\|\nabla^{k}\left(\frac{\varrho-\Theta}{1+\varrho} \nabla\right)\right\|^{2} .
\end{aligned}
$$

By $(26)_{1}$, we integrate by parts to obtain

$$
\begin{aligned}
-\int \partial_{t} \nabla^{k} u \cdot \nabla \nabla^{k} \mathrm{e}= & -\frac{d}{d t} \int \nabla^{k} u \cdot \nabla \nabla^{k} \mathrm{Q}+\int \nabla^{k} u \cdot \nabla \nabla^{k} \mathrm{e}_{t} \\
= & -\frac{d}{d t} \int \nabla^{k} u \cdot \nabla \nabla^{k} \mathrm{Q}+\int \nabla^{k} \operatorname{div} u \\
& \cdot\left[\nabla^{k} \operatorname{div} u+\nabla^{k} \operatorname{div}(\mathrm{e} u)\right] \\
& \leq-\frac{d}{d t} \int \nabla^{k} u \cdot \nabla \nabla^{k} \mathrm{Q}+\left\|\nabla^{k+1} u\right\|^{2}+\left\|\nabla^{k+1}(\mathrm{\varrho} u)\right\|^{2} \\
& \leq-\frac{d}{d t} \int \nabla^{k} u \cdot \nabla \nabla^{k} \mathrm{Q}+\left\|\nabla^{k+1} u\right\|^{2}+\delta\left\|\nabla^{k+1}(\mathrm{\varrho}, u)\right\|^{2},
\end{aligned}
$$

where we have used the product estimates (17) of Lemma 10 to estimate

$$
\left\|\nabla^{k+1}(\mathrm{\varrho} u)\right\| \leqslant\|\mathrm{\varrho}\|_{L^{\infty}}\left\|\nabla^{k+1} u\right\|+\left\|\nabla^{k+1} \mathrm{\varrho}\right\|\|u\|_{L^{\infty}} \leqslant \delta\left\|\nabla^{k+1}(\mathrm{\varrho}, u)\right\| .
$$

By Lemmas 10 and 11, we have

$$
\begin{gathered}
\left\|\nabla^{k}(u \cdot \nabla u)\right\| \lesssim\|u\|_{L^{\infty}}\left\|\nabla^{k+1} u\right\|+\left\|\nabla^{k} u\right\|_{L^{6}}\|\nabla u\|_{L^{3}} \leq \delta\left\|\nabla^{k+1} u\right\|, \\
\left\|\nabla^{k}\left(\frac{\mathrm{\varrho}-\Theta}{1+\varrho} \nabla \mathrm{\varrho}\right)\right\| \leqslant\|(\mathrm{\varrho}, \Theta)\|_{L^{\infty}}\left\|\nabla^{k+1} \mathrm{\varrho}\right\| \\
\quad+\left\|\nabla^{k}\left(\frac{\varrho-\Theta}{1+\varrho}\right)\right\|_{L^{6}}\|\nabla \mathrm{\varrho}\|_{L^{3}} \\
\leq \delta\left\|\nabla^{k+1}(\varrho, \Theta)\right\| .
\end{gathered}
$$

Plugging (63)-(66) into (62), we deduce (60). 
Finally, we collect all the dissipation estimates for $(\mathrm{\varrho}, u$, $\Theta)$ in Lemmas 17-19 to derive the following energy inequality with the minimum derivative counts.

Lemma 20. Let $N \geq 3$ and $T>0$. Then, there exists an energy functional $\mathscr{E}_{l}^{N}(t)$, which is equivalent to $\left\|\nabla^{l}(\mathrm{Q}, u, \Theta)(t)\right\|_{H^{N-l}}^{2}$, such that for any $t \in[0, T]$ and $0 \leq l \leq N-1$,

$\frac{d}{d t} \mathscr{E}_{l}^{N}(t)+\left\|\nabla^{l+1} \varrho(t)\right\|_{H^{N-l-1}}^{2}+\left\|\nabla^{l} u(t)\right\|_{H^{N-l}}^{2}+\left\|\nabla^{l+1} \Theta(t)\right\|_{H^{N-l}}^{2} \leq 0$,

under the assumption of

$$
\sup _{0 \leq t \leq T}\|(\mathrm{Q}, u, \Theta)(t)\|_{H^{3}}<\delta \ll 1, \quad \text { if } N=3
$$

or

$$
\sup _{0 \leq t \leq T}\|(\mathrm{\varrho}, u, \Theta)(t)\|_{H^{4}}<\delta \ll 1, \quad \text { if } N \geq 4
$$

Proof. Let $N \geq 3$ and $0 \leq l \leq N-1$. Summing up (29) of Lemma 17 from $k=l$ to $k=N-1$ and adding the resulting identity to (37) of Lemma 18 , since $\delta$ is small, we obtain

$$
\begin{aligned}
& \frac{d}{d t}\left[\left\|\nabla^{l}(\varrho, u, \Theta)(t)\right\|_{H^{N-l-1}}^{2}+\int \mathfrak{F}^{N}(t)\right] \\
& \quad+C_{1}\left(\left\|\nabla^{l} u(t)\right\|_{H^{N-l}}^{2}+\left\|\nabla^{l+1} \Theta(t)\right\|_{H^{N-l}}^{2}\right) \\
& \leq C_{2} \delta\left\|\nabla^{l+1} \varrho(t)\right\|_{H^{N-l-1}}^{2} .
\end{aligned}
$$

Summing up (60) of Lemma 19 from $k=l$ to $\mathrm{k}=N-1$, we obtain

$$
\begin{aligned}
& \frac{d}{d t} \int \sum_{k=l}^{N-1} \nabla^{k} u \cdot \nabla \nabla^{k} \mathrm{\varrho}+C_{3}\left\|\nabla^{l+1} \mathrm{\varrho}(t)\right\|_{H^{N-l-1}}^{2} \\
& \quad \leq C_{4}\left(\left\|\nabla^{l} u(t)\right\|_{H^{N-l}}^{2}+\left\|\nabla^{l+1} \Theta(t)\right\|_{H^{N-l-1}}^{2}\right) .
\end{aligned}
$$

Multiplying (71) by $2 C_{2} \delta / C_{3}$ and then adding it to (70), since $\delta$ is small, we deduce

$$
\begin{aligned}
& \frac{d}{d t}\left[\left\|\nabla^{l}(\mathrm{\varrho}, u, \Theta)(t)\right\|_{H^{N-l-1}}^{2}+\int \mathfrak{F}^{N}(t)+\frac{2 C_{2} \delta}{C_{3}} \int \sum_{k=l}^{N-1} \nabla^{k} u \cdot \nabla \nabla^{k} \mathrm{\varrho}\right] \\
& \quad+C_{5}\left(\left\|\nabla^{l+1} \mathrm{\varrho}(t)\right\|_{H^{N-l-1}}^{2}+\left\|\nabla^{l} u(t)\right\|_{H^{N-l}}^{2}+\left\|\nabla^{l+1} \Theta(t)\right\|_{H^{N-l}}^{2}\right) \\
& \quad \leq 0 .
\end{aligned}
$$

We define

$$
\begin{aligned}
\mathscr{E}_{l}^{N}(t):= & \frac{1}{C_{5}}\left[\left\|\nabla^{l}(\mathrm{\varrho}, u, \Theta)(t)\right\|_{H^{N-l-1}}^{2}+\int \mathfrak{F}^{N}(t)\right. \\
& \left.+\frac{2 C_{2} \delta}{C_{3}} \int \sum_{k=l}^{N-1} \nabla^{k} u \cdot \nabla \nabla^{k} \mathrm{\varrho}\right] .
\end{aligned}
$$

Note that

$\mathfrak{F}^{N}(t)=\frac{1+\Theta}{1+\varrho}\left|\nabla^{N} \varrho\right|^{2}+(1+\varrho)\left|\nabla^{N-1} \operatorname{div} u\right|^{2}+\left|\nabla^{N-1} \operatorname{curl} u\right|^{2}+\frac{1+\varrho}{1+\Theta}\left|\nabla^{N} \Theta\right|^{2}$.

By (28) and (56), since $\delta$ is small, we can deduce from (73) to (74) that there exists a positive constant $c$ such that for any $t \in[0, T]$,

$$
\frac{1}{c}\left\|\nabla^{l}(\varrho, u, \Theta)(t)\right\|_{H^{N-l}}^{2} \leq \mathscr{E}_{l}^{N}(t) \leq c\left\|\nabla^{l}(\varrho, u, \Theta)(t)\right\|_{H^{N-l}}^{2} .
$$

Hence, the proof of Lemma 20 is completed.

\section{Global Solution}

In this section, we will prove the existence and uniqueness of the global solution, namely, Theorem 1 . We first record the local solution (cf. [52]).

Proposition 21 (local-in-time solution). Let $l=3$ or 4 . Assume that $\left(\mathrm{\varrho}_{0}, u_{0}, \Theta_{0}\right) \in H^{l}$ and $\inf _{x \in \mathbb{R}^{3}}\left\{\mathrm{\varrho}_{0}(x)+1\right\}>0$. Then, there exists a constant $T>0$ such that the Cauchy problem (26) admits a unique solution $(\varrho, u, \Theta)(t) \in \mathscr{E}\left(0, T ; H^{l}\right)$ satisfying

$$
\left\{\begin{array}{l}
\inf _{x \in \mathbb{R}^{3}, 0 \leq t \leq T}\{\mathrm{\varrho}(x, t)+1\}>0, \\
\sup _{0 \leq t \leq T}\|(\varrho, u, \Theta)(t)\|_{H^{l}} \leq C_{1}\left\|\left(\mathrm{\varrho}_{0}, u_{0}, \Theta_{0}\right)\right\|_{H^{l}},
\end{array}\right.
$$

where $C_{1}>1$ is some fixed constant. Here,

$$
\begin{aligned}
\mathscr{E}\left(0, T ; H^{l}\right) & :=\{(\varrho, u, \Theta)(x, t):(\varrho, u)(x, t) \\
& \in C^{0}\left(0, T ; H^{l}\right) \cap C^{l}\left(0, T ; H^{l-1}\right), \Theta(x, t) \\
& \left.\in C^{0}\left(0, T ; H^{l}\right) \cap C^{l}\left(0, T ; H^{l-2}\right)\right\} .
\end{aligned}
$$

Then, we construct the a priori estimates by using the energy estimates given in Lemma 20.

Proposition 22 (a priori estimates). Let $N \geq 3$ and $T>0$. Assume that for some sufficiently small $\delta>0$,

$$
\sup _{0 \leq t \leq T}\|(\mathrm{\varrho}, u, \Theta)(t)\|_{H^{3}}<\delta, \quad \text { if } N=3
$$




$$
\sup _{0 \leq t \leq T}\|(\mathrm{Q}, u, \Theta)(t)\|_{H^{4}}<\delta, \quad \text { if } N \geq 4 \text {. }
$$

Then, we have for any $t \in[0, T]$ and $3 \leq \ell \leq N$,

$$
\begin{aligned}
& \|(\varrho, u, \Theta)(t)\|_{H^{\ell}}+\left(\int_{0}^{t}\left(\|\nabla \mathrm{\varrho}(\tau)\|_{H^{\ell-1}}^{2}+\|(u, \nabla \Theta)(\tau)\|_{H^{\ell}}^{2}\right) d \tau\right)^{1 / 2} \\
& \quad \leq C_{2}\left\|\left(\mathrm{\varrho}_{0}, u_{0}, \Theta_{0}\right)\right\|_{H^{\ell}},
\end{aligned}
$$

where $C_{2}>1$ is some fixed constant.

Proof. Let $N \geq 3$ and $3 \leq \ell \leq N$. Letting $l=0$ and $N=\ell$ in (67) of Lemma 20, we obtain

$$
\frac{d}{d t} \mathscr{E}_{0}^{\ell}(t)+\|\nabla \mathrm{Q}(t)\|_{H^{\ell-1}}^{2}+\|(u, \nabla \Theta)(t)\|_{H^{\ell}}^{2} \leq 0 .
$$

Integrating (81) in time, we obtain

$$
\mathscr{E}_{0}^{\ell}(t)+\int_{0}^{t}\left(\|\nabla \mathrm{\varrho}(\tau)\|_{H^{\ell-1}}^{2}+\|(u, \nabla \Theta)(\tau)\|_{H^{\ell}}^{2}\right) d \tau \leq \mathscr{E}_{0}^{\ell}(0) .
$$

Letting $l=0$ and $N=\ell$ in (75), we obtain for any $t \in[0, T]$ and some $c>0$,

$$
\frac{1}{c}\|(\varrho, u, \Theta)(t)\|_{H^{\ell}}^{2} \leq \mathscr{E}_{0}^{\ell}(t) \leq c\|(\varrho, u, \Theta)(t)\|_{H^{\ell}}^{2}
$$

We immediately deduce (80) from (82) to (83).

Finally, we perform a continuous argument to extend the local solution given in Proposition 21 to the global one. We only consider the case $N \geq 4$. It is similar for $N=3$.

Let $N \geq 4$. Assume $\left(\varrho_{0}, u_{0}, \Theta_{0}\right) \in H^{N}$ satisfying

$$
\left\|\left(\mathrm{\varrho}_{0}, u_{0}, \Theta_{0}\right)\right\|_{H^{4}}<\frac{\delta}{C_{1} C_{2}},
$$

where $C_{1}>1, C_{2}>1, \delta>0$ are given by Proposition 21 and Proposition 22. Since

$$
\left\|\left(\mathrm{Q}_{0}, u_{0}, \Theta_{0}\right)\right\|_{H^{4}}<\frac{\delta}{C_{1}}
$$

by Proposition 21, there exists a constant $T_{1}>0$ such that the Cauchy problem (26) has a unique local solution

$$
(\varrho, u, \Theta)(t) \in \mathscr{E}\left(0, T_{1} ; H^{4}\right),
$$

which satisfies

$$
\|(\varrho, u, \Theta)(t)\|_{H^{4}} \leq C_{1}\left\|\left(\mathrm{\varrho}_{0}, u_{0}, \Theta_{0}\right)\right\|_{H^{4}}<\delta, \quad \forall t \in\left[0, T_{1}\right] .
$$

By (87) and Proposition 22, we obtain for any $t \in\left[0, T_{1}\right]$ and $3 \leq \ell \leq N$,

$$
\|(\mathrm{\varrho}, u, \Theta)(t)\|_{H^{\ell}} \leq C_{2}\left\|\left(\mathrm{\varrho}_{0}, u_{0}, \Theta_{0}\right)\right\|_{H^{\ell}},
$$

which, together with (84), implies

$$
(\varrho, u, \Theta)\left(T_{1}\right) \in H^{4}, \quad\left\|(\mathrm{\varrho}, u, \Theta)\left(T_{1}\right)\right\|_{H^{4}}<\frac{\delta}{C_{1}} .
$$

Then, choosing $T_{1}>0$ as the new initial time instant, by Proposition 21 again, we obtain that the Cauchy problem (26) has a unique local solution

$$
(\varrho, u, \Theta)(t) \in \mathscr{E}\left(T_{1}, 2 T_{1} ; H^{4}\right),
$$

such that

$\|(\mathrm{\varrho}, u, \Theta)(t)\|_{H^{4}} \leq C_{1}\left\|(\mathrm{\varrho}, u, \Theta)\left(T_{1}\right)\right\|_{H^{4}}<\delta, \quad \forall t \in\left[T_{1}, 2 T_{1}\right]$.

From the above, we have proven that the Cauchy problem (26) has a unique local solution

$$
(\mathrm{\varrho}, u, \Theta)(t) \in \mathscr{E}\left(0,2 T_{1} ; H^{4}\right),
$$

such that

$$
\|(\mathrm{\varrho}, u, \Theta)(t)\|_{H^{4}}<\delta, \quad \forall t \in\left[0,2 T_{1}\right] .
$$

By (93) and Proposition 22, we obtain for any $t \in\left[0,2 T_{1}\right]$ and $3 \leq \ell \leq N$,

$$
\|(\varrho, u, \Theta)(t)\|_{H^{e}} \leq C_{2}\left\|\left(\varrho_{0}, u_{0}, \Theta_{0}\right)\right\|_{H^{\ell}},
$$

which, together with (84) again, implies

$$
(\mathrm{\varrho}, u, \Theta)\left(2 T_{1}\right) \in H^{4},\left\|(\mathrm{\varrho}, u, \Theta)\left(2 T_{1}\right)\right\|_{H^{4}}<\frac{\delta}{C_{1}} .
$$

By repeating the above procedures, we can extend the local solution to the global one only if $\left(\mathrm{\varrho}_{0}, u_{0}, \Theta_{0}\right) \in H^{N}(N$ $\geq 4)$ satisfying that $\left\|\left(\varrho_{0}, u_{0}, \Theta_{0}\right)\right\|_{H^{4}}$ is suitably small, as (84). So, we can choose $\delta_{0}=\delta / C_{1} C_{2}$ in Theorem 1. Hence, the proof of Theorem 1 is completed.

\section{Time-Decay Rates}

In this section, we shall derive the time-decay rates (9) in Theorem 2. We first show that the negative Sobolev or Besov norms of the solution $(\varrho, u, \Theta)(t)$ can be controlled by the initial data. 
Lemma 23. Under the assumptions of Theorem 1 , if further $\left(\mathrm{\varrho}_{0}, u_{0}, \Theta_{0}\right) \in \dot{H}^{-s}$ for some $s \in[0,3 / 2)$, or $\left(\mathrm{\varrho}_{0}, u_{0}, \Theta_{0}\right) \in \dot{B}_{2, \infty}^{-s}$ for some $s \in(0,3 / 2]$, then for all $t \geq 0$,

$$
\|(\mathrm{Q}, u, \Theta)(t)\|_{\dot{H}^{-s}} \leq C_{0}
$$

or

$$
\|(\varrho, u, \Theta)(t)\|_{\dot{B}_{2, \infty}^{-s}} \leq C_{0} .
$$

Proof. As with [29], Theorem 1.2, (1.7)-(1.8), we omit the details.

To derive the time-decay rates of the solution and its higher-order derivatives, we prove the following differential inequality with respect to time.

Lemma 24. Let $N \geq 3$. Under the assumptions of Theorem 1, it holds that for all $t \geq 0$ and $0 \leq l \leq N-1$,

$$
\frac{d}{d t} \mathscr{E}_{l}^{N}(t)+\left\|\nabla^{l+1} \mathrm{\varrho}(t)\right\|_{H^{N-l-1}}^{2}+\left\|\nabla^{l} u(t)\right\|_{H^{N-l}}^{2}+\left\|\nabla^{l+1} \Theta(t)\right\|_{H^{N-l}}^{2} \leq 0
$$

where

$$
\mathscr{E}_{l}^{N}(t) \sim\left\|\nabla^{l}(\varrho, u, \Theta)(t)\right\|_{H^{N-l}}^{2}
$$

Proof. It follows from Lemma 20 and Theorem 1.

Now, we can use Lemmas 23 and 24 to prove the timedecay rates (9) and (10) in Theorem 2.

For $0 \leq l \leq N-1$, by Lemmas 15 and 16 , we have

$$
\begin{aligned}
& \left\|\nabla^{l+1} f\right\| \gtrsim\|f\|_{\dot{H}^{-s}}^{-(1 /(l+s))}\left\|\nabla^{l} f\right\|^{1+(1 /(l+s))}, \\
& \left\|\nabla^{l+1} f\right\| \gtrsim\|f\|_{\dot{B}_{2, \infty}^{-s}}^{-(1 /(l+s))}\left\|\nabla^{l} f\right\|^{1+(1 /(l+s))} .
\end{aligned}
$$

Combining Lemma 23 with (100) and (101), we have

$$
\left\|\nabla^{l+1}(\varrho, \Theta)\right\| \geq C_{0}\left\|\nabla^{l}(\varrho, \Theta)\right\|^{1+(1 /(l+s))}
$$

This together with (8) infers for $0 \leq l \leq N-1$,

$$
\begin{gathered}
\left\|\nabla^{l+1} \mathrm{\varrho}(t)\right\|_{H^{N-l-1}}^{2}+\left\|\nabla^{l} u(t)\right\|_{H^{N-l}}^{2}+\left\|\nabla^{l+1} \Theta(t)\right\|_{H^{N-l}}^{2} \\
\geq C_{0}\left(\left\|\nabla^{l}(\varrho, u, \Theta)\right\|_{H^{N-l}}^{2}\right)^{1+(1 /(l+s))} .
\end{gathered}
$$

From the differential inequality (98) of Lemma 24, we obtain for $0 \leq l \leq N-1$,

$$
\frac{d}{d t} \mathscr{E}_{l}^{N}(t)+C_{0}\left(\mathscr{E}_{l}^{N}(t)\right)^{1+(1 /(l+s))} \leq 0
$$

Solving the above inequality directly, we get for $0 \leq l \leq$ $N-1$,

$$
\mathscr{E}_{l}^{N}(t) \leq C_{0}(1+t)^{-(l+s)}
$$

By (99), we have for $0 \leq l \leq N-1$,

$$
\left\|\nabla^{l}(\mathrm{\varrho}, u, \Theta)(t)\right\|_{H^{N-l}}^{2} \leq C_{0}(1+t)^{-(l+s)}
$$

Higher decay of the velocity $u$. Referring to (51), we know that the velocity $u$ satisfies

$$
u_{t}+u=-\nabla \varrho-\nabla \Theta-u \cdot \nabla u+\frac{\varrho-\Theta}{1+\varrho} \nabla \varrho .
$$

Let $N \geq 3$ and $0 \leq l \leq N-2$. Applying $\nabla^{l}$ to (107), multiplying the resulting identity by $\nabla^{l} u$ and integrating over $\mathbb{R}^{3}$, by Hölder's inequality, we obtain

$\frac{1}{2} \frac{d}{d t} \int\left|\nabla^{l} u\right|^{2}+\left\|\nabla^{l} u\right\|^{2} \leqslant\left\|\nabla^{l}\left(\nabla \varrho, \nabla \Theta, u \cdot \nabla u, \frac{\varrho-\Theta}{1+\varrho} \nabla \varrho\right)\right\|\left\|\nabla^{l} u\right\|$,

which infers

$$
\begin{aligned}
\frac{d}{d t}\left\|\nabla^{l} u\right\|^{2}+\left\|\nabla^{l} u\right\|^{2} & \lesssim\left\|\nabla^{l}\left(\nabla \mathrm{\varrho}, \nabla \Theta, u \cdot \nabla u, \frac{\mathrm{\varrho}-\Theta}{1+\varrho} \nabla \mathrm{\varrho}\right)\right\|^{2} \\
& \leq\left\|\nabla^{l+1}(\mathrm{\varrho}, \Theta)\right\|^{2}+\|(\mathrm{\varrho}, u, \Theta)\|_{H^{2}}^{2}\left\|\nabla^{l+1}(\mathrm{\varrho}, u, \Theta)\right\|^{2} \\
& \leq C_{0}(1+t)^{-(l+1+s)}
\end{aligned}
$$

by Cauchy's inequality, Lemmas 10 and 11, and (106). Applying Gronwall's inequality to (109), we obtain for $0 \leq l$ $\leq N-2$,

$$
\begin{aligned}
\left\|\nabla^{l} u(t)\right\|^{2} & \leq\left\|\nabla^{l} u_{0}\right\|^{2} e^{-t}+C_{0} \int_{0}^{t} e^{-(t-\tau)}(1+\tau)^{-(l+1+s)} d \tau \\
& \leq C_{0}(1+t)^{-(l+1+s)}
\end{aligned}
$$

Thus, we can deduce the decay rates (9) and (10) from (106) and (110). Hence, we complete the proof of Theorem 2.

\section{Data Availability}

The data that support the findings of this study are available from the corresponding author upon reasonable request.

\section{Conflicts of Interest}

The authors declare that they have no conflict of interest.

\section{Acknowledgments}

The first author was partially supported by the NSF of China (No. 11501143) and the Doctoral Research Project of 
Guizhou Normal University (No. GZNUD[2017]27). The second author was partially supported by Guangdong Provincial Pearl River Talents Program (No. 2017GC010407), Guangdong Province Basic and Applied Basic Research Fund (Nos. 2021A1515010235 and 2020B1515310002), Guangzhou City Basic and Applied Basic Research Fund (No. 202102020436), NSF of China (Nos. 11701264 and 11971179), and Science and Technology Program of Guangzhou (No. 2019050001).

\section{References}

[1] L. Hsiao and T. Luo, "Nonlinear diffusive phenomena of solutions for the system of compressible adiabatic flow through porous media," Journal of Differential Equations, vol. 125, no. 2, pp. 329-365, 1996.

[2] L. Hsiao and R. Pan, "Initial boundary value problem for the system of compressible adiabatic flow through porous media," Journal of Differential Equations, vol. 159, no. 1, pp. 280-305, 1999.

[3] P. Marcati and R. Pan, "On the diffusive profiles for the system of compressible adiabatic flow through porous media," SIAM Journal on Mathematical Analysis, vol. 33, no. 4, pp. 790826, 2001.

[4] C. M. Dafermos and R. Pan, "Global BV solutions for the psystem with frictional damping," SIAM Journal on Mathematical Analysis, vol. 41, no. 3, pp. 1190-1205, 2009.

[5] L. Hsiao, T. Luo, and T. Yang, "Global BV solutions of compressible Euler equations with spherical symmetry and damping," Journal of Differential Equations, vol. 146, no. 1, pp. 203-225, 1998.

[6] X. X. Ding, G. Q. Chen, and P. Z. Luo, "Convergence of the fractional step Lax-Friedrichs scheme and Godunov scheme for the isentropic system of gas dynamics," Communications in Mathematical Physics, vol. 121, no. 1, pp. 63-84, 1989.

[7] S. Geng and F. Huang, " $L^{1}$-convergence rates to the Barenblatt solution for the damped compressible Euler equations," Journal of Differential Equations, vol. 266, no. 12, pp. 7890-7908, 2019.

[8] F. Huang, P. Marcati, and R. Pan, "Convergence to the Barenblatt solution for the compressible Euler equations with damping and vacuum," Archive for Rational Mechanics and Analysis, vol. 176, no. 1, pp. 1-24, 2005.

[9] F. Huang and R. Pan, "Convergence rate for compressible Euler equations with damping and vacuum," Archive for Rational Mechanics and Analysis, vol. 166, no. 4, pp. 359376, 2003.

[10] F. Huang and R. Pan, "Asymptotic behavior of the solutions to the damped compressible Euler equations with vacuum," Journal of Differential Equations, vol. 220, no. 1, pp. 207-233, 2006.

[11] F. Huang, R. Pan, and Z. Wang, " $L^{1}$ convergence to the Barenblatt solution for compressible Euler equations with damping," Archive for Rational Mechanics and Analysis, vol. 200, no. 2, pp. 665-689, 2011.

[12] L. Hsiao, Quasilinear Hyperbolic Systems and Dissipative Mechanisms, World Scientific Publishing Co., Inc., River Edge, NJ, 1997.

[13] L. Hsiao and T.-P. Liu, "Convergence to nonlinear diffusion waves for solutions of a system of hyperbolic conservation laws with damping," Communications in Mathematical Physics, vol. 143, no. 3, pp. 599-605, 1992.
[14] R. Pan and K. Zhao, "Initial boundary value problem for compressible Euler equations with damping," Indiana University Mathematics Journal, vol. 57, no. 5, pp. 2257-2282, 2008.

[15] R. Pan and K. Zhao, "The 3D compressible Euler equations with damping in a bounded domain," Journal of Differential Equations, vol. 246, no. 2, pp. 581-596, 2009.

[16] P. Marcati and M. Mei, "Convergence to nonlinear diffusion waves for solutions of the initial boundary problem to the hyperbolic conservation laws with damping," Quarterly of Applied Mathematics, vol. 58, no. 4, pp. 763-784, 2000.

[17] K. Nishihara and T. Yang, "Boundary effect on asymptotic behaviour of solutions to the $p$-system with linear damping," Journal of Differential Equations, vol. 156, no. 2, pp. 439458, 1999.

[18] K. Nishihara, "Convergence rates to nonlinear diffusion waves for solutions of system of hyperbolic conservation laws with damping," Journal of Differential Equations, vol. 131, no. 2, pp. 171-188, 1996.

[19] K. Nishihara, W. Wang, and T. Yang, " $L_{p}$-convergence rate to nonlinear diffusion waves for $p$-system with damping," Journal of Differential Equations, vol. 161, no. 1, pp. 191218, 2000.

[20] H. Zhao, "Convergence to strong nonlinear diffusion waves for solutions of $p$-system with damping," Journal of Differential Equations, vol. 174, no. 1, pp. 200-236, 2001.

[21] L. Hsiao and D. Serre, "Global existence of solutions for the system of compressible adiabatic flow through porous media," SIAM Journal on Mathematical Analysis, vol. 27, no. 1, pp. 7077, 1996.

[22] T. Li and K. Zhao, "Analysis of non-isentropic compressible Euler equations with relaxation," Journal of Differential Equations, vol. 259, no. 11, pp. 6338-6367, 2015.

[23] R. Pan, "Darcy's law as long-time limit of adiabatic porous media flow," Journal of Differential Equations, vol. 220, no. 1, pp. 121-146, 2006.

[24] Q. Chen and Z. Tan, "Time decay of solutions to the compressible Euler equations with damping," Kinetic \& Related Models, vol. 7, no. 4, pp. 605-619, 2014.

[25] D. Fang and J. Xu, "Existence and asymptotic behavior of $C^{1}$ solutions to the multi-dimensional compressible Euler equations with damping," Nonlinear Analysis: Theory, Methods \& Applications, vol. 70, no. 1, pp. 244-261, 2009.

[26] J. Liao, W. Wang, and T. Yang, " $L^{p}$ convergence rates of planar waves for multi-dimensional Euler equations with damping," Journal of Differential Equations, vol. 247, no. 1, pp. 303329, 2009.

[27] Y. Liu and W. Wang, "Well-posedness of the IBVP for 2-D Euler equations with damping," Journal of Differential Equations, vol. 245, no. 9, pp. 2477-2503, 2008.

[28] T. C. Sideris, B. Thomases, and D. Wang, "Long time behavior of solutions to the 3D compressible Euler equations with damping," Communications in Partial Differential Equations, vol. 28, no. 3-4, pp. 795-816, 2003.

[29] Z. Tan and Y. Wang, "Global solution and large-time behavior of the $3 D$ compressible Euler equations with damping," Journal of Differential Equations, vol. 254, no. 4, pp. 1686-1704, 2013.

[30] Z. Tan and G. Wu, "Large time behavior of solutions for compressible Euler equations with damping in $\mathbb{R}^{3}$," Journal of Differential Equations, vol. 252, no. 2, pp. 1546-1561, 2012. 
[31] W. Wang and T. Yang, "The pointwise estimates of solutions for Euler equations with damping in multi-dimensions," Journal of Differential Equations, vol. 173, no. 2, pp. 410-450, 2001.

[32] W. Wang and T. Yang, "Existence and stability of planar diffusion waves for 2-D Euler equations with damping," Journal of Differential Equations, vol. 242, no. 1, pp. 40-71, 2007.

[33] R. Wei, Y. Li, and Z.-A. Yao, "Global existence and convergence rates of solutions for the compressible Euler equations with damping," Discrete \& Continuous Dynamical Systems $B$, vol. 25, no. 8, pp. 2949-2969, 2020.

[34] Z. Wu and W. Wang, "Large time behavior and pointwise estimates for compressible Euler equations with damping," Science China Mathematics, vol. 58, no. 7, pp. 1397-1414, 2015.

[35] X. Yang and W. Wang, "The suppressible property of the solution for three-dimensional Euler equations with damping," Nonlinear Analysis: Real World Applications, vol. 8, no. 1, pp. 53-61, 2007.

[36] T. Luo and H. Zeng, "Global existence of smooth solutions and convergence to Barenblatt solutions for the physical vacuum free boundary problem of compressible Euler equations with damping," Communications on Pure and Applied Mathematics, vol. 69, no. 7, pp. 1354-1396, 2016.

[37] H. Zeng, "Global resolution of the physical vacuum singularity for three-dimensional isentropic inviscid flows with damping in spherically symmetric motions," Archive for Rational Mechanics and Analysis, vol. 226, no. 1, pp. 33-82, 2017.

[38] H. Zeng, "Almost global solutions to the three-dimensional isentropic inviscid Flows with damping in a physical vacuum around Barenlatt solutions," Archive for Rational Mechanics and Analysis, vol. 239, no. 1, pp. 553-597, 2021.

[39] Q. Chen, Z. Tan, and G. Wu, "Time decay rates for the equations of the compressible heat-conductive flow through porous media," Journal of Differential Equations, vol. 259, no. 9, pp. 4707-4731, 2015.

[40] G. Wu, Z. Tan, and J. Huang, "Global existence and large time behavior for the system of compressible adiabatic ow through porous media in $\mathbb{R}^{3}$," Journal of Differential Equations, vol. 255, no. 5, pp. 865-880, 2013.

[41] Z. Wu and X. Miao, "Large time behavior for the system of compressible adiabatic flow through porous media in $\mathbb{R}^{3}$," Journal of Mathematical Analysis and Applications, vol. 472, no. 1, pp. 112-132, 2019.

[42] Y. Zhang and G. Wu, "The 3D non-isentropic compressible Euler equations with damping in a bounded domain," Chinese Annals of Mathematics, Series B, vol. 37, no. 6, pp. 915-928, 2016.

[43] F. Wu, "Global existence and nonlinear diffusion of classical solutions to nonisentropic Euler equations with damping in bounded domain," Journal of Hyperbolic Differential Equations, vol. 13, no. 1, pp. 147-179, 2016.

[44] Y. Guo and Y. Wang, "Decay of dissipative equations and negative Sobolev spaces," Communications in Partial Differential Equations, vol. 37, no. 12, pp. 2165-2208, 2012.

[45] A. Matsumura and T. Nishida, "The initial value problem for the equations of motion of compressible viscous and heatconductive fluids," Proceedings of the Japan Academy, Series A, Mathematical Sciences, vol. 55, no. 9, pp. 337-342, 1979.

[46] L. Nirenberg, "On elliptic partial differential equations," Annali della Scuola Normale Superiore di Pisa. Classe di Scienze, vol. 13, no. 3, pp. 115-162, 1959.
[47] N. Ju, "Existence and uniqueness of the solution to the dissipative 2D quasigeostrophic equations in the Sobolev space," Communications in Mathematical Physics, vol. 251, no. 2, pp. 365-376, 2004.

[48] Y. Wang, C. Liu, and Z. Tan, "A generalized Poisson-NernstPlanck-Navier-Stokes model on the fluid with the crowded charged particles: derivation and its well-posedness," SIAM Journal on Mathematical Analysis, vol. 48, no. 5, pp. 31913235, 2016.

[49] Y. Wang, C. Liu, and Z. Tan, "Well-posedness on a new hydrodynamic model of the fluid with the dilute charged particles," Journal of Differential Equations, vol. 262, no. 1, pp. 68-115, 2017.

[50] E. M. Stein, "Singular integrals and differentiability properties of functions," in Princeton mathematical series, no. 30, Princeton University press, Princeton, N.J., 1970.

[51] V. Sohinger and R. M. Strain, "The Boltzmann equation, Besov spaces, and optimal time decay rates in $\mathbb{R}_{x}^{n}$," Advances in Mathematics, vol. 261, pp. 274-332, 2014.

[52] A. Matsumura and T. Nishida, "The initial value problem for the equations of motion of viscous and heat-conductive gases," Kyoto Journal of Mathematics, vol. 20, no. 1, pp. 67-104, 1980. 